study investigating use of high-dose human albumin (ALB) for the treatment of acute ischemic stroke. ALB has previously been shown to have strong neuroprotective properties in rodent models of stroke.

A total of 82 patients with acute ischemic stroke participated in the study. Six successive cohorts received an infusion of $25 \%$ ALB within $16 \mathrm{~h}$ of stroke onset, with dose tiers increasing from $0.34 \mathrm{~g} / \mathrm{kg}$ to $2.05 \mathrm{~g} / \mathrm{kg}$. Fortytwo of the patients also received intravenous tissue plasminogen activator-the current standard of care for acute ischemic stroke.

In addition to regular monitoring of vital signs, patients underwent regular cardiac evaluation and were given brain CT scans and chest X-rays after treatment. Patients were assigned NIHSS (NIH stroke scale) scores at baseline, at regular intervals during the first $72 \mathrm{~h}$ after treatment, at discharge and 1 month after discharge. At 3 months post-discharge, efficacy outcomes were determined using the NIHSS scale, modified Rankin scale (mRS) and Barthel Index.

After adjustment for the effects of tissue plasminogen activator, the probability of a good outcome at 3 months (defined as an NIHSS score of 0-1 and/or an mRS score of 0-1) for the three highest ALB doses was found to be $81 \%$ higher than for the three lowest doses, and 95\% higher than for comparable patients from the NINDS rt-PA Stroke Study. The only adverse event was mild to moderate pulmonary edema, which occurred in $13 \%$ of subjects and was successfully managed with diuretics.

On the basis of these results, a large phase III trial is now underway, and is expected to conclude in 2010.

Original articles Ginsberg MD et al. (2006) The ALIAS Pilot Trial: a dose-escalation and safety study of albumin therapy for acute ischemic stroke-I: physiological responses and safety results. Stroke 37: 2100-2106

Palesch YY et al. (2006) The ALIAS Pilot Trial: a doseescalation and safety study of albumin therapy for acute ischemic stroke-II: neurologic outcome and efficacy analysis. Stroke 37: 2107-2114

\section{Cerebral artery thrombi show similar structural composition regardless of etiology}

Researchers from the David Geffen School of Medicine at the University of California, Los Angeles, have carried out a histopathological study of thromboemboli retrieved from the cerebral artery networks of patients with acute ischemic stroke. A mechanical retrieval device allowed the researchers to study emboli that would previously have been inaccessible.

Marder et al. removed thrombi from 25 patients shortly after the defined onset of stroke symptoms (mean delay between symptom onset and retrieval $6.2 \pm 3.1 \mathrm{~h}$ ). Most thromboemboli were retrieved in fragments, although removal as a single mass was achieved in $36 \%$ of cases. Only thromboemboli $<3 \mathrm{~mm}$ wide reached the middle cerebral artery, whereas emboli extracted from the internal carotid artery reached a maximum width of $5 \mathrm{~mm}$. Each thrombus sample had a unique histological appearance, despite the majority being composed of a complicated pattern of platelet and fibrin areas combined with deposits of nucleated cells and erythrocytes. This structural composition is consistent with clinical trial data indicating that both antiplatelet and anticoagulant agents are beneficial in stroke prevention. No relationship was found between thrombus histology and presumed cardiopathic or arteriopathic etiology or occlusion location, and no calcific deposits or cholesterol crystals were observed in any of the samples.

In addition to the 25 patients in whom thrombus extraction was successful, 29 patients underwent unsuccessful thrombus retrieval. The success of thrombus extraction was negatively correlated with patient age, but no relationship was found with sex, the timing of the retrieval procedure, stroke subtype or site of the thromboembolism.

Original article Marder VJ et al. (2006) Analysis of thrombi retrieved from cerebral arteries of patients with acute ischemic stroke. Stroke 37: 2086-2093 